\title{
The WASP transit surveys
}

\section{Andrew Collier Cameron ${ }^{1}$, Don Pollacco ${ }^{2}$, Coel Hellier ${ }^{3}$, Richard West ${ }^{4}$, the WASP Consortium and the SOPHIE \& CORALIE Planet-Search Teams}

\author{
${ }^{1}$ SUPA, School of Physics and Astronomy, University of St Andrews, North Haugh, \\ St Andrews, Fife KY16 9SS, UK \\ email: acc4@st-and.ac.uk \\ ${ }^{2}$ ARC, School of Mathematics \& Physics, Queen's University, Belfast, BT7 1NN, UK \\ ${ }^{3}$ Astrophysics Group, Keele University, Staffordshire, ST5 5BG, UK \\ ${ }^{4}$ Department of Physics and Astronomy, University of Leicester, Leicester, LE1 7RH, UK
}

\begin{abstract}
The WASP consortium operates two wide-field camera arrays in the northern (La Palma) and southern (Sutherland) hemispheres. Each installation comprises a robotic mount bearing 8 cameras, forming a mosaic with a field of view of 30 degrees in declination by 1 hour in right ascension. Here we describe the system performance, observing strategy, candidate selection procedures and follow-up campaigns, and the yield and physical characteristics of the 16 planets discovered in the course of the survey to date.
\end{abstract}

\section{Introduction}

The discovery of HD 209458b, the first transiting exoplanet, provided the first confirmation that Jupiter-mass planets in close orbits about their host stars could have radii and densities comparable to the gas-giant planets of our own solar system (Charbonneau et al. 2000, Henry et al. 2000). Since then, the number of known transiting planets has grown to several dozen. A few of the very brightest have been discovered in the same way as HD 209458b, through intensive photometric followup of known radial-velocity planets at times of inferior conjunction. The remainder, however, are beginning to fulfil the promise of the prototype STARE instrument that captured the first transits of HD 209458b: to use small-aperture, wide-field imaging systems based on commercial optics of modest cost, to survey the entire sky for the brightest transiting exoplanets. The motivation is to discover gas-giant planets around stars bright enough for radial-velocity confirmation and mass measurements to be obtained on $2 \mathrm{~m}$-class telescopes equipped with high-precision radial-velocity spectrometers. The discovery observations themselves reveal the mass and density of a newly-discovered planet, providing key insights into its internal structure. The other motivation for such wide, shallow surveys is to discover planets orbiting stars bright enough to enable detailed followup studies of their atmospheric transmission and emission spectra. The remarkable advances reported elsewhere in this volume from studies of this kind attest to the success of this strategy.

The Wide-Angle Search for Planets (WASP) has yielded 16 new transiting planets since its inaugural observing season in 2004. Here we describe the hardware, observing strategy and data analysis, and give a brief summary of trends seen in the physical parameters of the planets found so far. We examine the detection threshold and discuss ways in which further improvements in sensitivity may be gained. 


\section{Camera arrays and observing strategy}

The WASP camera arrays are located at the Observatorio del Roque de los Muchachos on La Palma, Canary Islands, and at Sutherland Observatory in South Africa. The instruments are described in detail by Pollacco et al. (2006). Each comprises an array of eight cameras on a robotic equatorial mount. Each camera consists of a $200 \mathrm{~mm} \mathrm{f} / 1.8$ Canon lenses backed by an Andor CCD array of $2048^{2} 13.5 \mu$ m pixels, with 3 -stage Peltier cooling to $-50 \mathrm{C}$. The field of view of each camera is 7.8 degrees square; that of the entire array covers approximately one hour in RA by 30 degrees in declination. Observations are conducted in a raster pattern, cycling from field centres located 3 to 4 hours east of the meridian to 3 or 4 hours west. Every hour a field is dropped in the west, and a new one picked up in the east. During the first two years of full-time operation, starting in 2006 May, the transit-search fields have been primarily located in bands centred at declinations $\pm 30^{\circ}$. Fields are observed provided they are accessible for at least $4 \mathrm{~h}$ each night. The observing season for a given object typically spans 120 to 150 nights.

\section{Pipeline processing and decorrelation}

The data are reduced using the SuperWASP pipeline (Pollacco et al. (2006)). Science frames are bias-subtracted and flat-fielded. An automated field recognition algorithm identifies the objects on the frame with their counterparts in the TYCHO- 2 catalogue and establishes an astrometric solution with an RMS precision of 0.1 to 0.2 pixel. Aperture photmetry is then carried out at the positions on each CCD image of all objects in the USNO-B1.0 catalogue with magnitudes brighter than 15.0. The formal variance of the flux of each star in the image is computed from the photon counts in the stellar aperture and the surrounding sky annulus.

After correction for primary and secondary extinction, the zero-point for each image is tied to a network of local secondary standards in each field. The resulting instrumental fluxes are transformed via a colour equation relating instrumental magnitudes to TYCHO$2 \mathrm{~V}$ magnitudes. The resulting fluxes are stored in the SuperWASP Data Archive at the University of Leicester.

Transit searches are conducted on all stars brighter than $V=13.0$ in each complete dataset uploaded to the archive at the end of the observing season for the field concerned. Patterns of correlated systematic error are identified and removed using the SysRem algorithm of Tamuz, Mazeh \& Zucker (2005) and the Trend-Filtering Algorithm (TFA) of Kovács, Bakos \& Noyes (2005). At this stage the photometric variances are augmented by an additional systematic variance that accounts for the actual scatter in the measured fluxes about the final decorrelated solution for each frame. The maximum-likelihood algorithm for computing this additional variance is described by Collier Cameron et al. (2006). The long-term RMS scatter of the decorrelated SuperWASP light curve of a nonvariable star is $0.004 \mathrm{mag}$ at $\mathrm{V}=9.5$, degrading to $0.01 \mathrm{mag}$ at $\mathrm{V}=12.0$ as shown in Fig. 3 of Collier Cameron et al. (2006).

\section{Candidate identification and winnowing}

Transit searches are conducted on a field-by-field basis following decorrelation, using a hybrid search algorithm described by Collier Cameron et al. (2006). We use a modified version of the Box Least-Squares (BLS) algorithm (Kovacs et al. 2002). The initial BLS search is conducted using a coarse search grid. The epoch, period, depth and duration of each of the five strongest signals in the BLS periodogram is refined using a 
Newton-Raphson method based on the analytically-differentiable transit profile model of Protopapas, Jimenez \& Alcock (2005). We also test for ellipsoidal variability in the out-of-transit light curve, and use the reduced proper-motion method to flag probable giants with anomalously low proper motions for their 2MASS magnitudes and colours.

The BLS algorithm finds many candidates whose transit depths and durations are inconsistent with expectations for gas-giant planets. Collier Cameron et al. (2007b) use the 2MASS colours of transit candidates to estimate the stellar mass and radius, making the assumption that the star is on the main sequence. We use the best BLS solution as the starting point for a more detailed Markov-chain Monte Carlo (MCMC) derivation of the stellar mass and radius, the companion radius and the impact parameter of the transit. This uses the model of Mandel \& Agol (2002) in the small-planet approximation, using the 4-coefficient non-linear limb-darkening model of Claret (2000). We impose a Bayesian prior on the stellar mass and radius, by minimizing the statistic

$$
S=\chi^{2}+Q
$$

where

$$
Q=\frac{\left(m_{*}-m_{0}\right)^{2}}{\sigma_{m}^{2}}+\frac{\left(r_{*}-r_{0}\right)^{2}}{\sigma_{r}^{2}} .
$$

Here $m_{0}, r_{0}$ are the main-sequence mass and radius most appropriate to the 2MASS $J-H$ colour, with estimated variances $\sigma_{m}^{2}$ and $\sigma_{r}^{2}$. In many grazing stellar binaries and in cases where a late-type giant is blended with a nearby eclipsing binary, the transit duration is inconsistent with a main-sequence stellar radius. This drives the best-fitting stellar mass $m_{*}$ and radius $r_{*}$ to values that are inconsistent with each other and with their expected main-sequence values. A large value of the statistic $Q>5$ indicates that the object is probably an astrophysical false positive. We also use the posterior probability distribution from the MCMC analysis to determine the probability that the companion's radius is less than a specified threshold - usually 1.5 Jupiter radii.

Candidates that pass these tests are subjected to visual inspection to weed out spurious signals, and are then scheduled for followup transit photometry using a number of telescopes accessible to consortium members. The goal is to eliminate objects which pass the MCMC tests but which nonetheless turn out to be faint eclipsing binaries located within the 48-arcsec photometric aperture used by the WASP pipeline. These higherprecision transit profiles are used with MCMC to refine the system parameters, and may often unmask grazing stellar binaries that have survived earlier winnowing.

\section{Radial-velocity followup}

Radial-velocity followup of the surviving candidates is performed using the SOPHIE spectrograph (Bouchy et al. 2006) on the 1.93-m telescope at Haute-Provence, and the CORALIE spectrograph (Queloz et al. 2000) on the 1.2-m Leonard Euler Telescope operated at La Silla by the Geneva Observatory. Our present winnowing strategy leads to a success rate of approximately one planet per five or six candidates selected for radialvelocity followup. Double-lined binaries and very fast rotators can be rejected after a single observation. Narrow, single-lined stellar binaries usually become apparent after a second-epoch radial-velocity measurement. Objects showing radial-velocity variations of planet-like amplitude are followed intensively until 10 or so measurements have been obtained around the orbit. At this stage we eliminate hierarchical triples by testing for correlation of the span bisector with the radial velocity (Queloz et al. 2001). 


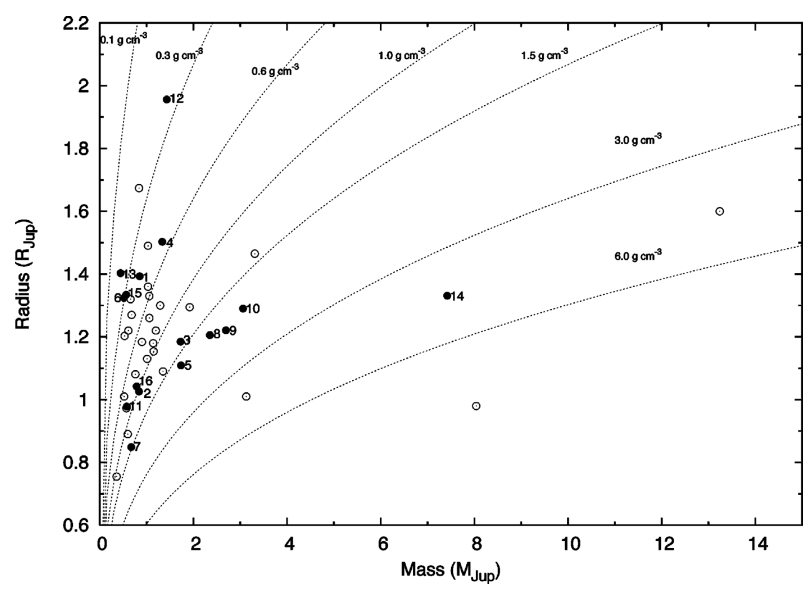

Figure 1. Mass-radius diagram for transiting exoplanets announced to 2008 May 19. Filled symbols denote WASP planets and are numbered in order of radial-velocity confirmation. Contours of constant density are superimposed.

\section{Planetary parameters}

In determining the physical parameters of a transiting planet and its host star we use high-resolution spectroscopy to establish the stellar effective temperature, surface gravity and metallicity. A simultaneous MCMC analysis of the light curve and radial-velocity curve establishes the stellar density directly from the transit profile via Kepler's 3rd law (Carter et al. 2008) . By comparing the star's location in the $\left(T_{\mathrm{eff}}, R / M^{1 / 3}\right)$ plane against isochrones and evolutionary tracks derived from the stellar evolution models of Girardi

Table 1. Physical parameters of the 16 WASP planets and host stars.

\begin{tabular}{c|c|c|c|c|c|c|c}
\hline WASP & $\begin{array}{c}M_{\text {planet }} \\
\left(M_{\text {Jup }}\right)\end{array}$ & $\begin{array}{c}R_{\text {planet }} \\
\left(R_{\text {Jup }}\right)\end{array}$ & $\begin{array}{c}P \\
(\text { days })\end{array}$ & $\begin{array}{c}a \\
(\mathrm{au})\end{array}$ & $\begin{array}{c}M_{*} \\
\left(M_{\odot}\right)\end{array}$ & $\begin{array}{c}R_{*} \\
\left(R_{\odot}\right)\end{array}$ & $\begin{array}{c}T_{\text {eff }} \\
(K)\end{array}$ \\
\hline 1 & $0.79 \pm 0.09$ & $1.44_{-0.04}^{+0.04}$ & 2.5200 & 0.0379 & $1.15 \pm 0.13$ & $1.45 \pm 0.06$ & $6110 \pm 45$ \\
2 & $0.88 \pm 0.08$ & $1.02_{-0.06}^{+0.06}$ & 2.1522 & 0.0310 & $0.86 \pm 0.08$ & $0.80 \pm 0.04$ & $5380 \pm 200$ \\
3 & $1.73 \pm 0.13$ & $1.18_{-0.06}^{+0.08}$ & 1.8468 & 0.0317 & $1.24 \pm 0.12$ & $1.22 \pm 0.07$ & $6330 \pm 200$ \\
4 & $1.35 \pm 0.11$ & $1.41_{-0.06}^{+0.06}$ & 1.3382 & 0.0239 & $1.01 \pm 0.10$ & $0.94 \pm 0.04$ & $5720 \pm 200$ \\
5 & $1.73 \pm 0.12$ & $1.11_{-0.04}^{+0.09}$ & 1.6284 & 0.0278 & $1.08 \pm 0.11$ & $1.05 \pm 0.05$ & $5880 \pm 200$ \\
6 & $0.52 \pm 0.05$ & $1.32_{-0.11}^{+0.10}$ & 3.3610 & 0.0446 & $1.04 \pm 0.12$ & $0.96 \pm 0.07$ & $5770 \pm 200$ \\
7 & $0.96 \pm 0.15$ & $0.92_{-0.04}^{+0.05}$ & 4.9547 & 0.0618 & $1.28 \pm 0.14$ & $1.24 \pm 0.06$ & $6400 \pm 100$ \\
8 & $2.35 \pm 0.17$ & $1.21_{-0.06}^{+0.19}$ & 8.1602 & 0.0822 & $1.11 \pm 0.11$ & $1.04 \pm 0.08$ & $5950 \pm 200$ \\
9 & $2.69 \pm 0.41$ & $1.22_{-0.05}^{+0.11}$ & 2.0000 & 0.0333 & $1.23 \pm 0.13$ & $1.04 \pm 0.05$ & $6120 \pm 200$ \\
10 & $2.96 \pm 0.22$ & $1.28_{-0.08}^{+0.08}$ & 3.0928 & 0.0371 & $0.70 \pm 0.08$ & $0.78 \pm 0.04$ & $4675 \pm 100$ \\
11 & $0.58 \pm 0.05$ & $0.98_{-0.07}^{+0.05}$ & 3.7225 & 0.0432 & $0.77 \pm 0.08$ & $0.79 \pm 0.04$ & $4800 \pm 100$ \\
12 & $1.42 \pm 0.11$ & $1.91_{-0.09}^{+0.13}$ & 1.0914 & 0.0235 & $1.44 \pm 0.15$ & $1.66 \pm 0.10$ & $6400 \pm 200$ \\
13 & $0.44 \pm 0.05$ & $1.40_{-0.28}^{+0.15}$ & 4.3532 & 0.0528 & $1.04 \pm 0.12$ & $1.54 \pm 0.20$ & $5800 \pm 100$ \\
14 & $7.73 \pm 0.50$ & $1.26_{-0.06}^{+0.08}$ & 2.2438 & 0.0358 & $1.32 \pm 0.13$ & $1.30 \pm 0.07$ & $6475 \pm 100$ \\
15 & $0.56 \pm 0.07$ & $1.33_{-0.10}^{+0.06}$ & 3.7521 & 0.0483 & $1.07 \pm 0.13$ & $1.38 \pm 0.07$ & $6130 \pm 200$ \\
16 & $0.76 \pm 0.06$ & $1.04_{-0.06}^{+0.05}$ & 3.1186 & 0.0417 & $1.00 \pm 0.10$ & $0.98 \pm 0.05$ & $5770 \pm 200$ \\
\hline
\end{tabular}

References:

${ }^{1}$ Collier Cameron et al. (2007a), Charbonneau et al. (2007), Shporer et al. (2007), Stempels et al. (2007);

${ }^{2}$ Collier Cameron et al. (2007a), Charbonneau et al. (2007); ${ }^{3}$ Pollacco et al. $(2008) ;{ }^{4}$ Wilson et al. (2008);

${ }^{5}$ Anderson et al. (2008); ${ }^{6}$ Final observations pending; ${ }^{7}$ Hellier et al. (2008); ${ }^{8}$ Queloz et al., in prep.; ${ }^{9}$ Final observations pending; ${ }^{10}$ Christian et al. (2008); ${ }^{11}$ West et al. 2008 , in prep.; ${ }^{12} \mathrm{Hebb}$ et al. 2008, in prep.;

${ }^{13}$ Skillen et al. 2008 , in prep.; ${ }^{14}$ Joshi et al. $(2008) ;{ }^{15}$ Final observations pending; ${ }^{16}$ Final observations pending. 


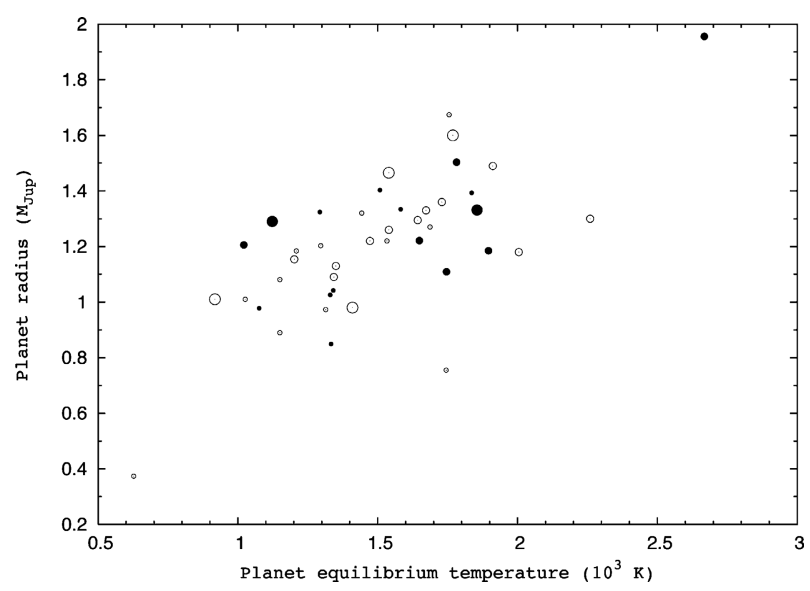

Figure 2. Radius-equilibrium temperature diagram for transiting exoplanets announced to 2008 May 19. Filled symbols denote WASP planets. Symbol sizes denote planet masses in the ranges $M_{p}<1.0 M_{\mathrm{Jup}}$ (small), $1.0 M_{\mathrm{Jup}}<M_{p}<1.0 M_{\mathrm{Jup}}$ (medium), and $M_{p}>3.0 M_{\mathrm{Jup}}$ (large).Planetary equilibrium temperatures are calculated assuming a blackbody model with zero albedo and isotropic re-radiation of the incident stellar power. The lack of planets in the lower part of the diagram results from incompleteness at small planet radii.

et al. (2000), we refine our estimates of the stellar mass and age. This new estimate of the stellar mass is fed back into the MCMC code as a Bayesian prior, ensuring a self-consistent set of parameters at the end of the process.

At the time of writing, radial-velocity studies of WASP candidates have led to the identification of 16 transiting planets, eight in each hemisphere. Their basic parameters are summarised in Table 1, and their masses and radii are plotted together with those of the other known transiting planets in Fig. 1. Among them, the most extreme objects are WASP-12b and WASP-14b. The former is in a 1.09-day orbit about a mid-F star, and has a radius nearly twice that of Jupiter. WASP-14b is a massive planet in an eccentric 2.2-day orbit (Joshi et al. 2008). Its mass is comparable to that of HAT-P-2b, but it orbits substantially closer to its host star, and has a markedly lower density.

In Fig. 2 we plot planetary radii against planetary blackbody equilibrium temperatures assuming zero albedo and complete redistribution of heat from the dayside to the nightside. The planetary equilibrium temperature is thus purely a function of the incident flux. We note that the upper boundary of the planet distribution in this plot shows an apparently linear increase in maximum planet radius with irradiating flux. There is, however, no obvious trend with planetary mass at a given equilibrium temperature.

\section{Detection threshold}

In Fig. 3 we plot the transit depths measured by the transit-search algorithm as a function of the host stars' $V$ magnitudes. Although the numbers are small, we see a rather weak dependence of minimum transit depth on $V$ magnitude. Around stars brighter than $V=11$ the detection threshold is less than 0.01 magnitude. The lack of deeper transits at the brighter magnitudes is interesting, and may reflect the predominance of relatively early-type stars in this magnitude range. Between $V=11.3$ and $V=12.3$ we begin to see an increase in the deeper transits expected of intrinsically fainter, later-type stars. The detectability of even the largest planets appears to fall dramatically beyond $V=12.5$. 


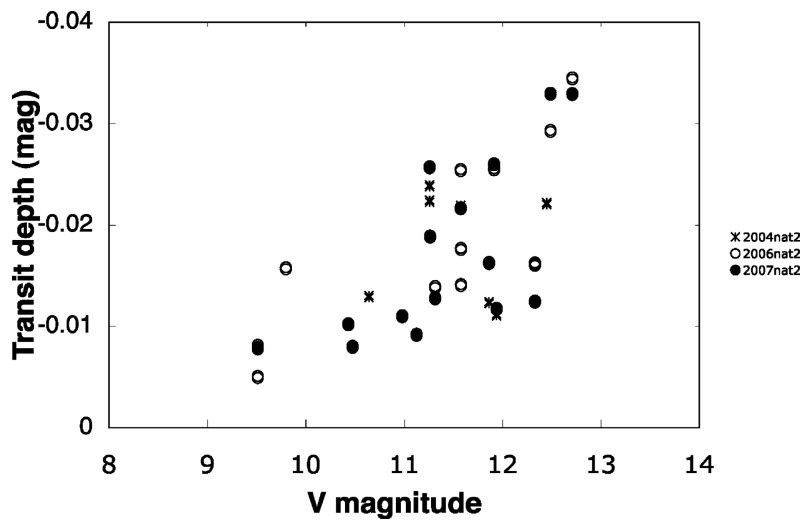

Figure 3. Transit depths versus $V$ magnitude for transiting planets detected in 2004, 2006 and 2007 observing seasons. The transit depths are raw estimates from the detection algorithm, so the same planet may be detected at slightly different depths in different seasons. The plot includes planets discovered by other teams, but which yielded detections qualifying them as transit candidates in the WASP survey regions.

\section{Outlook}

We are currently investigating a number of ways to deepen the survey volume and to push the detection threshold for planets orbiting around bright stars down toward Saturnlike and smaller radii. The camera lenses incorporate a $\mathrm{CaF}$ lens element whose refractive index is sufficiently temperature-sensitive to give a measurable correlation between the width of the point-spread function and the ambient temperature. We aim to eliminate this source of correlated error by active stabilisation of the lens temperature.

The WASP pipeline currently performs aperture photometry on the image frames. A recent feasibility study by Alsubai (2008) on the use of differential image analysis (DIA) on the fields containing WASP-7 and WASP-8 produced a substantial reduction in systematic errors. This study concluded that the use of DIA would yield the same RMS scatter as aperture photometry, in stars 0.7 magnitude fainter. This corresponds to an effective increase in the WASP survey volume by a factor 2.6. We are currently working towards implementation of DIA in the second-generation WASP data pipeline.

Finally we are conducting multiple-season period searches, in order to increase the detection probability in systems whose periods yield a relatively small number of transits in any single observing season. This will improve both the period completeness among shorter-period systems, and our sensitivity to planets in wider orbits.

\section{Acknowledgments}

The WASP Consortium consists of representatives from Queens University Belfast and the Universities of Keele, Leicester, St Andrews and The Open University, together with the ING (La Palma) and the IAC (Tenerife). The SuperWASP and WASP-S Cameras were constructed and operated with funds made available from Consortium Universities and PPARC/STFC. The WASP cameras are hosted by ING and SAAO (Sutherland), and we are grateful for their support and assistance. 


\section{References}

Alsubai, K., 2008, PhD thesis, University of St Andrews

Anderson, D. R., et al., 2008, MNRAS, 387, L4

Bouchy, F., The Sophie Team, 2006, tafp.conf, 319

Collier Cameron, A., et al., 2006, MNRAS, 373, 799

Collier Cameron, A., et al., 2007a, MNRAS, 375, 951

Collier Cameron, A., et al., 2007b, MNRAS, 380, 1230

Carter, J. A., Yee, J. C., Eastman, J., Gaudi, B. S., \& Winn, J. N., 2008, arXiv, 805, arXiv:0805.0238

Charbonneau, D., Brown, T. M., Latham, D. W., \& Mayor, M., 2000, ApJ, 529, L45

Charbonneau, D., Winn, J. N., Everett, M. E., Latham, D. W., Holman, M. J., Esquerdo, G. A., \& O'Donovan, F. T., 2007, ApJ, 658, 1322

Christian, D. J., et al., 2008, MNRAS, submitted (arXiv:0806.1482)

Claret A., 2000, A\&A, 363, 1081

Girardi, L., Bressan, A., Bertelli, G., \& Chiosi, C., 2000, A\&AS, 141, 371

Hellier, C., et al., 2008, ApJ, submitted (arXiv:0805.2600)

Henry, G. W., Marcy, G. W., Butler, R. P., \& Vogt, S. S., 2000, ApJ, 529, L41

Joshi, Y. C., et al., 2008, MNRAS, submitted (arXiv:0806.1478)

Kovács, G., Zucker, S., \& Mazeh, T., 2002, A\&A, 391, 369

Kovács, G., Bakos, G., \& Noyes, R. W., 2005, MNRAS, 356, 557

Mandel, K. \& Agol, E., 2002, ApJL, 580, L171

Pollacco, D. L., et al., 2006, PASP, 118, 1407

Pollacco, D., et al., 2008, MNRAS, 385, 1576

Protopapas, P., Jimenez, R., \& Alcock, C., 2005, MNRAS, 362, 460

Queloz, D., et al., 2000, A\&A, 354, 99

Queloz, D., et al., 2001, A\&A, 379, 279

Shporer, A., Tamuz, O., Zucker, S., \& Mazeh, T., 2007, MNRAS, 376, 1296

Stempels, H. C., Collier Cameron, A., Hebb, L., Smalley, B., \& Frandsen, S., 2007, MNRAS, 379,773

Tamuz, O., Mazeh, T., \& Zucker, S., 2005, MNRAS, 356, 1466

Wilson, D. M., et al., 2008, ApJ, 675, L113 